\title{
Recombination Monophosphoryl Lipid A Derived Vacosome for the Development of Preventive Cancer Vaccines
}

Ruoyu Cheng ${ }^{\dagger}$, Flavia Fontana ${ }^{\dagger}$, Junyuan Xiao ${ }^{\ddagger}$ Zehua Liu ${ }^{\dagger}$, Patrícia Figueiredo ${ }^{\dagger}$, Mohammad-Ali

Shahbazi $i^{\dagger} \|$, Shiqi Wang ${ }^{\dagger}$, Jing Jin ${ }^{\ddagger}$, Giulia Torrieri ${ }^{\dagger}$, Jouni T. Hirvonen ${ }^{\dagger}$, Hongbo Zhang ${ }^{\ddagger}$, Tongtong Chen", Wenguo Cui ${ }^{*, \downarrow}$, Yong Lu ${ }^{*, l}$ and Hélder A. Santos ${ }^{*, \dagger, \delta}$

${ }^{\dagger}$ Drug Research Program, Division of Pharmaceutical Chemistry and Technology, Faculty of Pharmacy, University of Helsinki, FI-00014 Helsinki, Finland

¥ Shanghai Key Laboratory for Prevention and Treatment of Bone and Joint Diseases, Shanghai Institute of Traumatology and Orthopaedics, Ruijin Hospital, Shanghai Jiao Tong University School of Medicine, 197 Ruijin Second Road, 200025 Shanghai, PR China

" Radiology Department, Ruijin Hospital, Shanghai Jiao Tong University School of Medicine, 197 Ruijin 2nd Road, Shanghai 200025, PR China.

II Department of Pharmaceutical Sciences Laboratory and Turku Center for Biotechnology, Åbo Akademi University, Turku FI-20520, Finland

|| Department of Pharmaceutical Nanotechnology, School of Pharmacy, Zanjan University of Medical Sciences, 45139-56184 Zanjan, Iran

${ }^{\delta}$ Helsinki Insititute of Life Science, HiLIFE, University of Helsinki, FI-00014 Helsinki, Finland

\section{*Corresponding authors}

wgcui80@ hotmail.com (W.Cui); 18917762053@163.com(Y.Lu); helder.santos@ helsinki.fi (H.A. Santos) 


\section{EXPERIMENTAL SECTION}

Materials. Monophosphoryl lipid A (MPLA) and DSPE-PEG-2000 were purchased from Avanti Polar Lipid, Inc. CD11c-FITC, CD80-PE, CD86-APC, CD3FITC, CD4-BV605, CD8-Percp cy5, CD45-BV421, FoxP3-PE, CD62L-Pecy7, CD44BV785, Zombie aqua fixable viability kit and cytometric bead array (CBA) were all purchased from BioLegend.

Cell Membrane Derivation. The cell membrane of 4T1 cells was harvested, following a previously published protocol. ${ }^{1}$ Briefly, 4T1 cells (ATCC CRL-2539) were detached by PBS-EDTA (Gibco, Thermo Fisher) and then washed with $1 \times$ PBS three times. Cells were then resuspended in hypotonic lysing buffer $(20 \mathrm{mM}$ of Tris- $\mathrm{HCl}, \mathrm{pH}$ 7.5, $10 \mathrm{mM}$ of $\mathrm{KCl}, 2 \mathrm{mM}$ of $\mathrm{MgCl}_{2}$ and 1 tablet protease inhibitor (Thermo Fisher) per $10 \mathrm{~mL}$ solution). After gradient centrifugation (20000 $\mathrm{g}$ for $20 \mathrm{~min}$ and $100000 \mathrm{~g}$ for 10 min, Optima MAX, Beckmann Coulter, USA), the pellet was collected for further application. Membrane content was quantified by a micro bicinchoninic acid assay (BCA) protein assay kit (Thermo Fisher) in the reference to a bovine serum albumin (BSA) standard. Approximately 10 million 4T1 cells were able to yield $0.8 \mathrm{mg}$ cell membranes (by protein weight).

Dendritic Cell Maturation Studies. BMDCs were collected from marrow cavities of femurs and tibias of BALB/c mice (4-6 weeks), as described elsewhere. ${ }^{2}$ BMDCs $\left(1 \times 10^{5}\right.$ cell per well in 24 well plate $)$ were treated with different amounts of MPLA, cell membrane and lipid materials for $24 \mathrm{~h}$. After treatment, BMDCs were costained with CD11c-FITC, CD80-PE, CD86-APC for the maturation assay by flow 
cytometry (BD, FACS LSR II).

Synthesis of Vacosome. MPLA-Lip was synthesized by the ethanol injection method. ${ }^{3}$ Basically, MPLA, DSPE-PEG-2000, EPC and cholesterol were dissolved in ethanol and then mixture was injected into deionized water (final concentration of MPLA $1 \mu \mathrm{g} / \mathrm{mL}$, EPC 7.81mg/ mL, DSPE-PEG-2000 $1 \mathrm{mg} / \mathrm{mL}$ and cholesterol 2.16 mg/mL in the MPLA@Lip solution).MPLA@Lip\#F was prepared by the same method, expect using DSPE-PEG (2000)-FITC. To prepare the vacosome, the same amount of MPLA, DSPE-PEG-2000, EPC, and cholesterol were dissolved in ethanol, and then the lipid solution was injected into cell membrane solution with constant stirring. Afterwards, the resulting solution was bath sonicated and physically extruded through a $200 \mathrm{~nm}$ polycarbonate membrane (Avanti Lipids) for 11 passes. Vacosome\#F was fabricated by the similar process, expect using DSPE-PEG (2000)-FITC. Additionally, DSPE-PEG (2000)-FITC, EPC and cholesterol were dissolved in ethanol and injected into deionized water to prepare Lip\#F.

Characterization of Vacosome. The hydrodynamic size and surface zetapotential of cell membrane (CM), MPLA@Lip and vacosome were measured with dynamic light scattering and electrophoretic mobility (Malvern Zetasizer, Malvern, UK), respectively. The long-term stability of vacosome was also investigated by DLS in the $1 \times \mathrm{PBS}$ at $4{ }^{\circ} \mathrm{C}$ without stirring. To examine the morphology, $\mathrm{CM}$, the MPLA@Lip and vacosome were dropped to copper grids and stained with uranyl acetate $(0.2 \mathrm{wt} \%)$ for $10 \mathrm{secs}$, then left drying at room temperature. Samples were visualized by transmission electron microscope (TEM, FEI). 
Cellular Uptake and Biocompatibility. To test the BMDCs' uptake, Lip\#F, MPLA@Lip\#F and vacosome\#F were co-incubated with BMDCs $\left(1 \times 10^{5}\right.$ cell per well in 24 well plate) for $3,6,24,48 \mathrm{~h}$. Then trypan blue (Thermo Fisher) was used to eliminate any influence caused by surface binding between the BMDCs and the nanoparticles. The uptake efficacy was evaluated by flow cytometry (BD, FACS LSR II).

For the biocompatibility tests, firstly the nanoparticles were co-cultured with BMDCs $\left(1 \times 10^{5}\right.$ cell per well in 24 well plate $)$ for 1,2 , and 3 days. Then the cell viability was assessed with CellTiter-Glo reagent assay (Promega Corporation), according to the manufacturer's instructions. BMDCs cultured with RPMI1640 medium (10 \% FBS), 1\% Triton X-100 and RPMI1640 medium (10\% FBS) with lipid materials (DSPEPEG2000, EPC and cholesterol) were used as negative, positive and lipid controls (NC, PC, and LC), respectively. The luminescence was recorded using a Varioskan Flash multimode reader (Thermo Fisher).

In Vitro Immune Activation. BMDCs maturation was analyzed by flow cytometry. BMDCs were treated with various formulation for 24,48 and $72 \mathrm{~h}$. After that, BMDCs were co-stained with CD11c-FITC, CD80-PE, CD86-APC for the maturation assay. For further immune activation test, firstly, BMDCs were co-cultured with different formulation for 3 days, then these cells were centrifuged (500g) and washed by $1 \times$ PBS for three times. Afterwards, these BMDCs cells were co-cultured with splenocytes (derived from a 4-6 weeks' old female BALB/c mice) for another 3 days. Finally, both BMDCs and splenocytes cells were added into the planted 4T1 cells 
for 24 h' incubation (4T1 cells were plated one day before BMDCs and splenocytes cells were added). Then, all suspensions were discarded, and each well was gently washed with $1 \times$ PBS for three times. The cell viability of 4T 1 was evaluated using the CellTiter-Glo reagent assay. Additionally, the morphology of the 4T1 cells were evaluated by microscope (Leica, DMi1).

In Vivo Immune Activation. Female BALB/c mice (4-6 weeks) were purchased from Shanghai Biomodel Organism Science \& Technology Development Co.,Ltd. Mice were divided into groups randomly. BALB/c mice were fed under the protocols approved by Shanghai Jiao Tong University Laboratory Animal Center. The animal experiments were in accordance with international ethics guidelines and the National Institutes of Health Guide, concerning the Care and Use of Laboratory Animals.

For the biocompatibility and the immune activation tests in vivo, different formulations (saline, CM, MPLA@Lip and vacosome) were subcutaneously injected into the mice on day 0,11 and 25 . Blood samples of mice were collected on day 7, 14 and 28, and then the sera were isolated for the further cytometric bead array, in order to evaluate the secretion of IL-12p70, TNF- $\alpha$, and IFN- $\gamma$, according to the manufacturer's instructions (LEGENDplex ${ }^{\mathrm{TM}}$-BioLegend). Additionally, the body-weight of mice with different treatments was recorded every two days. On day 28 , three mice were randomly chosen from each group and the kidney, heart, and liver were collected to evaluate the biocompatibility of vacosome in vivo by H\&E staining.

\section{Analysis of Different Subpopulation of $T$ Cells in Tumors and Spleens.}

$\mathrm{BALB} / \mathrm{c}$ mice were immunized three times with various formulations, as 
abovementioned ( 25 mice for each groups). 3 days after the last vaccination, 4 T1 cells $\left(2 \times 10^{5}\right.$ cell per mouse) were subcutaneously injected into the mice. The injected day was recorded as day 0 in the tumor stage. Then the tumor size ( 6 mice for each group) and the survival rate (10 mice for each group) of mice was recorded every two days. On day 14 , five mice from each group were selected to analyze the immunological profile in both tumor and spleen. Specifically, single-cell suspensions from the tumor and spleen were prepared, as described elsewhere. ${ }^{4}$ Flow cytometry (BD, FACS Celesta) was used to analyzed these cells, specifically, for the CD8+T cells, central memory $\mathrm{T}$ cells $\left(\mathrm{T}_{\mathrm{CM}}\right)$ and effector memory $\mathrm{T}$ cells $\left(\mathrm{T}_{\mathrm{EM}}\right)$ were marked as $\mathrm{CD} 3+\mathrm{CD} 8+\mathrm{CD} 62 \mathrm{~L}+\mathrm{CD} 44+$ and $\mathrm{CD} 3+\mathrm{CD} 8+\mathrm{CD} 62 \mathrm{~L}-\mathrm{CD} 44+$, respectively. For the CD4+T cells were further marked as regulatory $T$ cells $\left(\mathrm{T}_{\text {reg }}\right)(\mathrm{CD} 3+\mathrm{CD} 4+\mathrm{Foxp} 3+)$, and zombie aqua fixable viability kit was used to gate the living cells.

Statistical Analysis. To compare differences between the groups, one-way ANOVA with Tukey's multiple comparison test was used (IBM SPSS Statistics 25). *p $<0.05, * * \mathrm{p}<0.01$ and ${ }^{* * *} \mathrm{p}<0.001$ were considered statistically significant. Values are expressed as mean $\pm \mathrm{SD}$. 


\section{References}

(1) Fang, R. H.; Hu, C.-M. J.; Luk, B. T.; Gao, W.; Copp, J. A.; Tai, Y.; O’Connor, D. E.; Zhang, L. Cancer Cell Membrane-coated Nanoparticles for Anticancer Vaccination and Drug Delivery. Nano Lett. 2014, 14, 2181-2188.

(2) Han, X.; Wang, R.; Xu, J.; Chen, Q.; Liang, C.; Chen, J.; Zhao, J.; Chu, J.; Fan, Q.; Archibong, E.; Jiang, L.; Wang, C.; Liu, Z. In Situ Thermal Ablation of Tumors in Combination with Nano-adjuvant and Immune Checkpoint Clockade to Inhibit Cancer Metastasis and Recurrence. Biomaterials 2019, 224, 119490.

(3) Pons, M.; Foradada, M.; Estelrich, J. Liposomes Obtained by the Ethanol Injection Method. Int. J. Pharm. 1993, 95, 51-56.

(46) Chen, Q.; Chen, J.; Yang, Z.; Xu, J.; Xu, L.; Liang, C.; Han, X.; Liu, Z. Nanoparticle-Enhanced Radiotherapy to Trigger Robust Cancer Immunotherapy. Adv. Mater. 2019, 31, 1802228. 

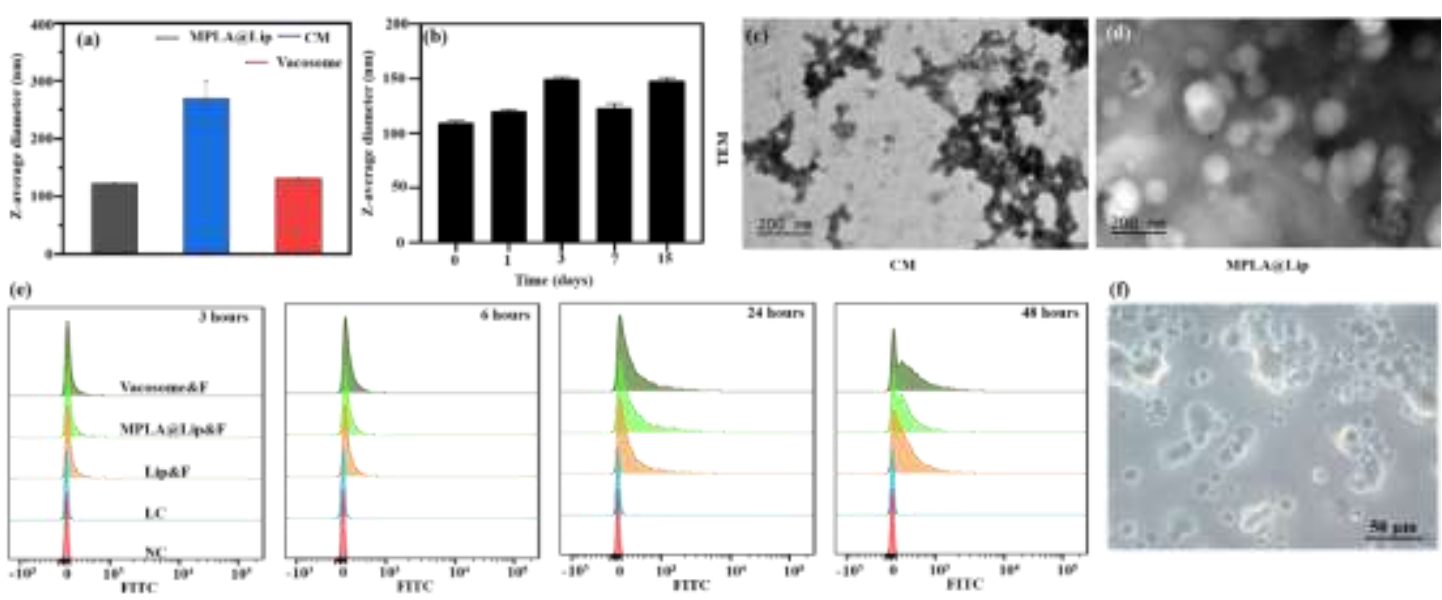

Figure S1. a) The Z-averages of MPLA@Lip, CM and vacosome. b) The Z-averages of vacosome for long-term stability investigation. TEM images of c) $\mathrm{CM}$ and d) MPLA@Lip. e) The cytometry results of FITC+BMDCs co-cultured with different formulation. f) The antitumor ability of T cells induced by the vacosome. The red arrow indicates that $4 \mathrm{~T} 1$ cells were surrounded by the $\mathrm{T}$ cells that were trying to kill the $4 \mathrm{~T} 1$ cells. 


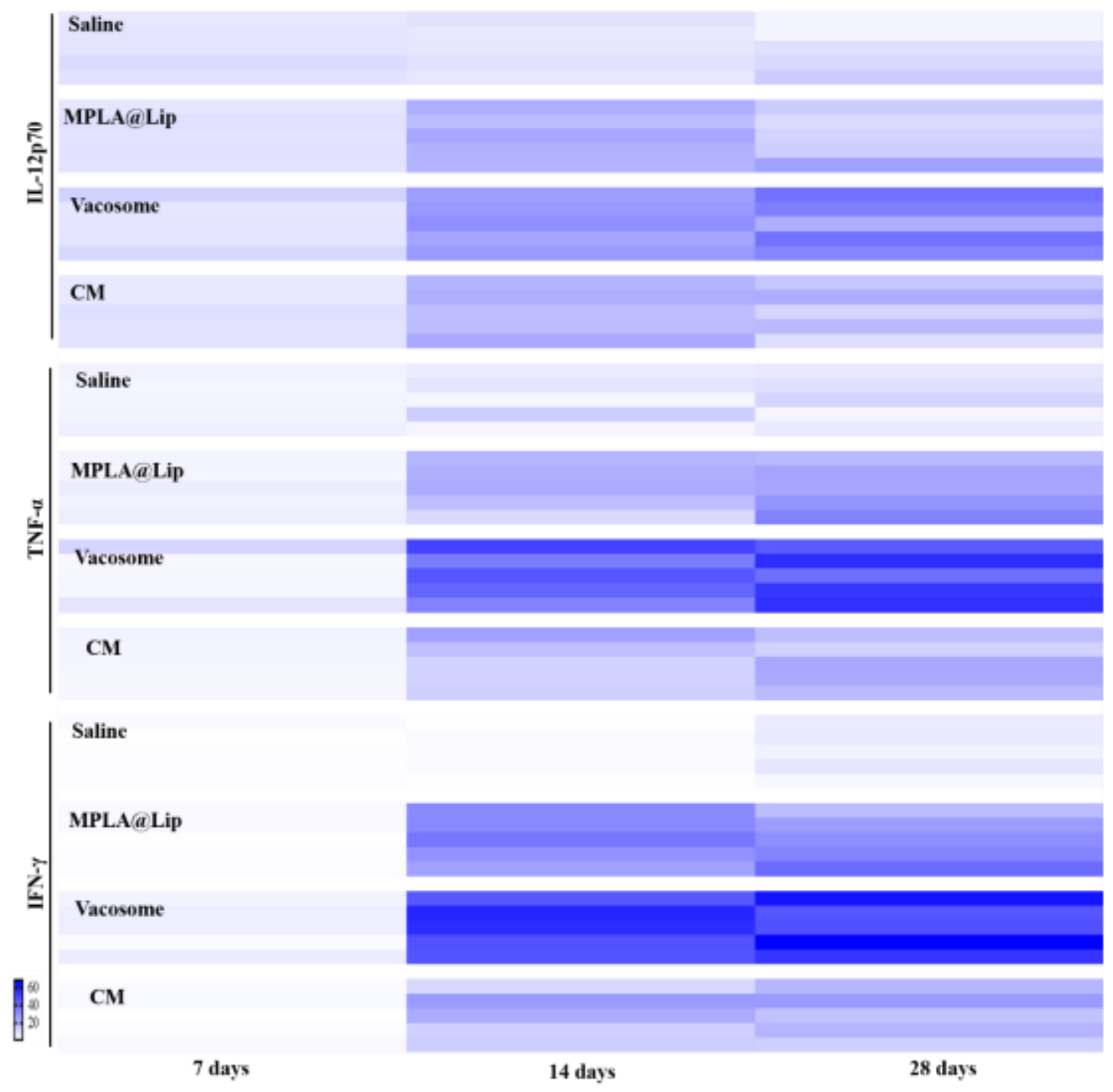

Figure S2. Heatmap illustrating cytokines concentrations at different preimmunization stages. 

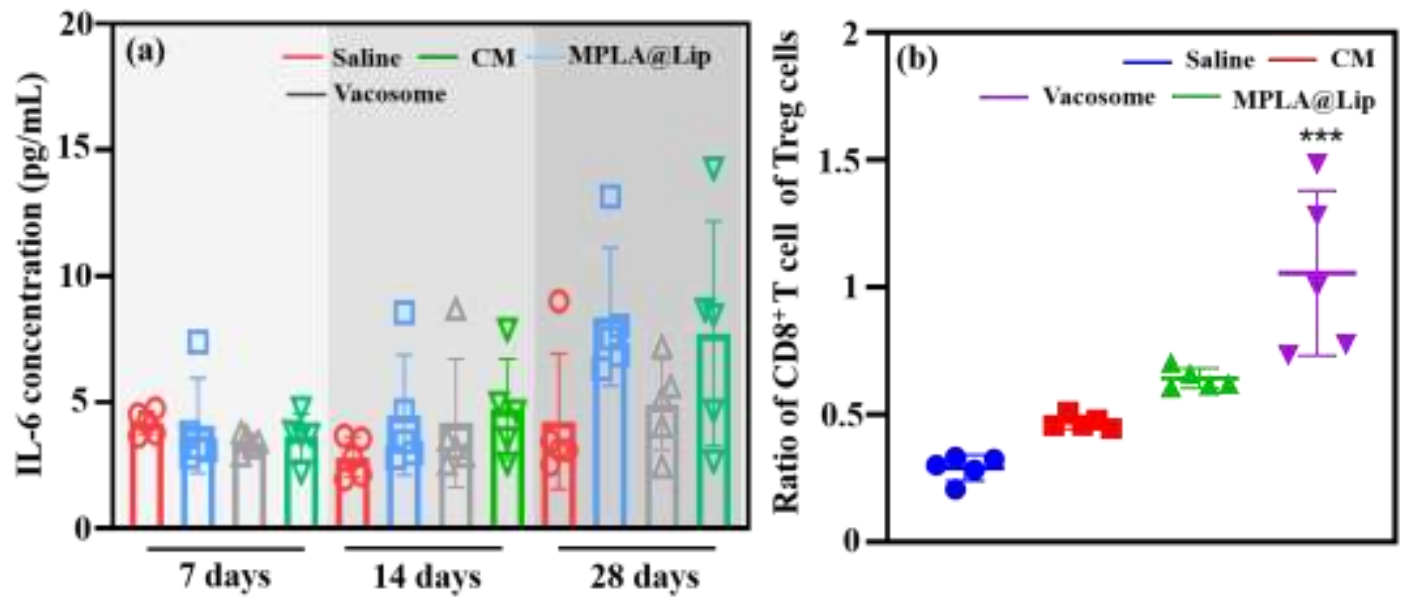

Figure S3. Immunological profiles. a) The concentration of IL-6 in the serum after different vaccinations. b) The ration of CD8+ T cells of Treg cells in the collected tumor tissues. 


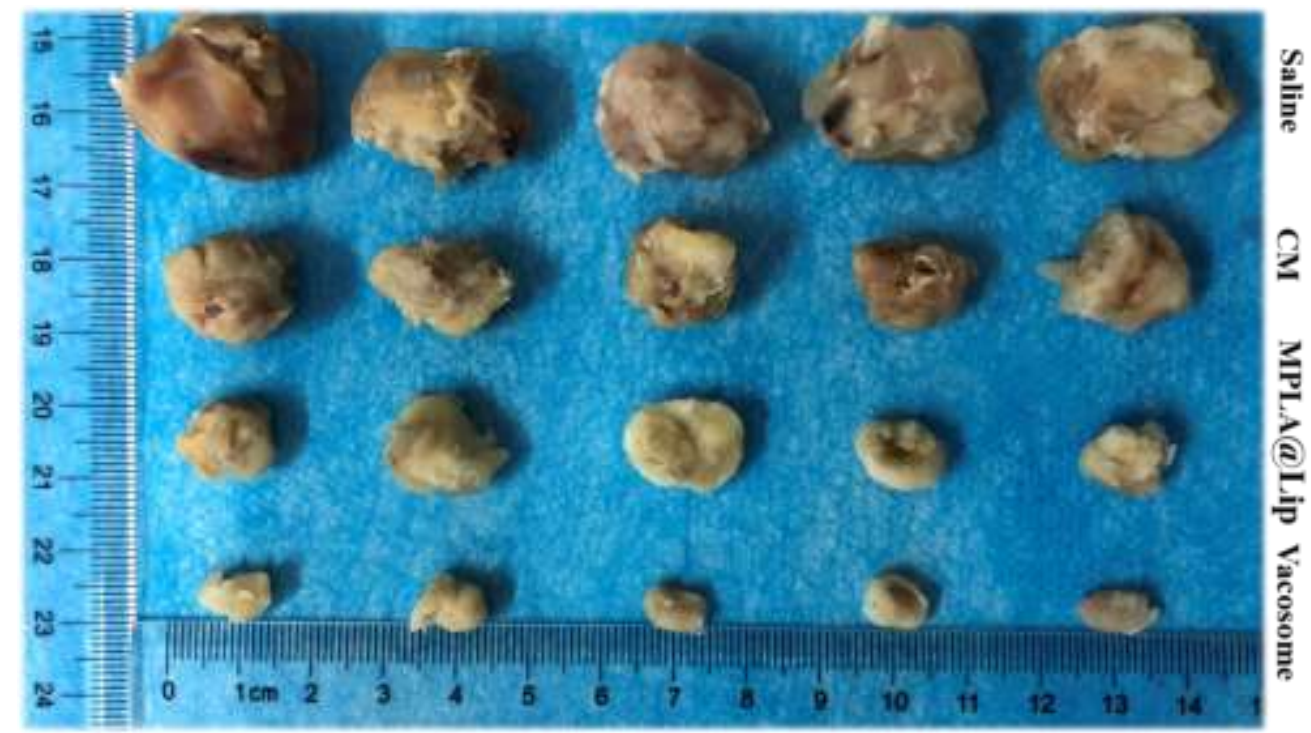

Figure S4. The collected tumor tissues in 4T1-bearing balb/c after 21 days' treatment with different formulation. 


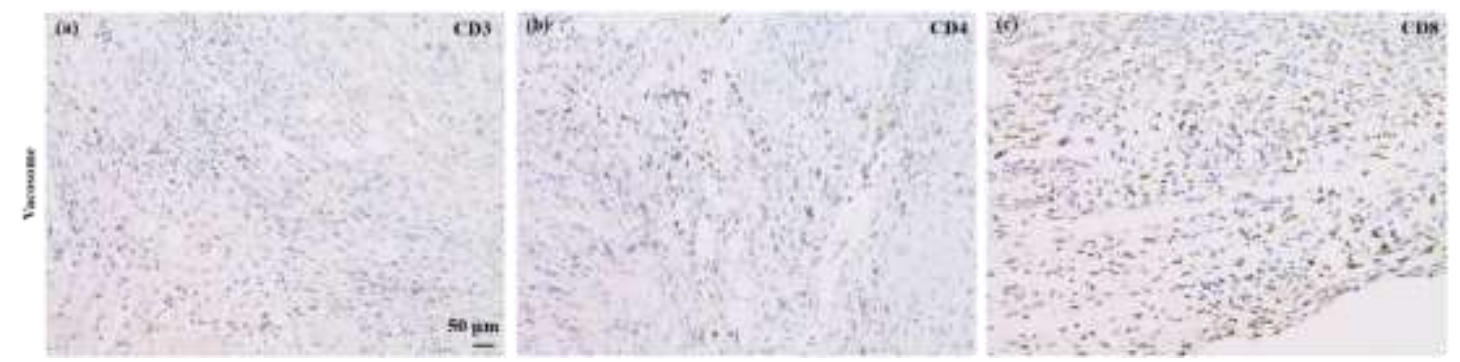

Figure S5. The immunohistochemistry staining of vacosome groups, a) $\mathrm{CD}^{+}$, b) $\mathrm{CD}^{+}$, and c) $\mathrm{CD}^{+} \mathrm{T}$ cells in the tumor tissue. 